\title{
Photon and Carrier Management Design for Nonplanar Thin-Film Copper Indium Gallium Selenide Photovoltaics
}

\author{
Colton R. Bukowsky ${ }^{\mathrm{a}, *}$, Jonathan Grandidier ${ }^{\mathrm{a}, \mathrm{b}}$, Katherine T. Fountaine ${ }^{\mathrm{a}, \mathrm{c}}$, Dennis M. Callahan ${ }^{\mathrm{a}, \mathrm{d}}$, Billy J. \\ Stanbery ${ }^{\mathrm{e}, \mathrm{f}}$, Harry A. Atwater ${ }^{\mathrm{a}, *}$ \\ ${ }^{a}$ California Institute of Technology, 1200 E. California Drive, Pasadena, California 91125, USA \\ ${ }^{b}$ Currently with the Jet Propulsion Laboratory, 4800 Oak Grove Dr., Pasadena, California 91109, USA \\ ${ }^{c}$ Currently with Northrup Grumman Aerospace Systems, One Space Park Dr., Redondo Beach, California 90278, USA \\ ${ }^{d}$ Currently with The Charles Stark Draper Laboratory, Inc., 555 Technology Square, Cambridge, MA 02139, USA \\ ${ }^{e}$ Helio Volt Corporation, 6301 E Stassney Ln, Austin, Texas 78744, USA \\ ${ }^{f}$ Currently with Siva Power, 5102 Calle del Sol, Santa Clara, California 95054, USA
}

\begin{abstract}
Nonplanar structured photovoltaic absorber design has potential to achieve high solar cell efficiency with significantly reduced material use. We report optoelectronic simulations that highlight photon and generated carrier management opportunities for improvement of thin film $\mathrm{Cu}\left(\mathrm{In}_{\mathrm{x}} \mathrm{Ga}_{1-\mathrm{x}}\right) \mathrm{Se}_{2}$ (CIGS) device performance. Structures realized via either self-assembly or patterning via nanoimprint lithography, and also a combination of both are predicted to exhibit significant increases in short circuit current density and open circuit voltage simultaneously. The structures investigated include: 1) self-assembled nonplanar structures that strongly scatter incident light and enhance carrier generation near regions of high electric potential, 2) lithographically-patterned embedded periodic dielectric structures, 3) planar dielectric layers that separate the CIGS absorber from the molybdenum back-contact via reduced-area contacts that minimize optical and electronic losses, 4) a combination of these for combined effects. We find that the self-assembled nonplanar CIGS cells with $700 \mathrm{~nm}$ planar equivalent thickness, combined with dielectric separation layers yield increases in short circuit current density and open circuit voltage up to $3.4 \mathrm{~mA} \mathrm{~cm}^{-2}$ and $29 \mathrm{mV}$, respectively. The absolute efficiency increases from $15.4 \%$ to $18.1 \%$, compared to the predicted efficiency for planar CIGS thin film cells of equivalent thickness. The addition of a single layer $\mathrm{MgF}_{2}$ anti-reflection coating brings the maximum predicted efficiency up to $19.7 \%$ for randomly textured devices.
\end{abstract}

Keywords: solar cells, thin film, light trapping, CIGS, nanotextured

\section{Introduction}

Thin-film solar cells have been researched as alternatives to crystalline silicon solar cells for decades due to high efficiencies greater than $20 \%$ and the intrinsic benefits of thicknesses on the order of microns, such mechanical flexibility and a variety of substrate options. Typically, CIGS photovoltaic devices are between 1-3 $\mu \mathrm{m}$ thick, allowing the majority of incident sunlight to be absorbed. Reducing the thickness of devices below $1 \mu \mathrm{m}$ is desirable to increase production throughput capacity while reducing the materials cost associated the rare-earth metal indium, which is also highly in demand for large area applications by the flat-panel display industry. Reducing thickness would also decrease the number of minority carriers lost to bulk recombination, thereby increasing the voltage of devices. 1] However sub-micron cells cannot absorb all incident photons in a traditional planar architecture, and the benefits

\footnotetext{
* Corresponding author

Email addresses: cb@caltech.edu (Colton R. Bukowsky), haa@caltech.edu (Harry A. Atwater)
}

of reduced bulk recombination are lost due to surface recombination at the back-contact. Here, we demonstrate that nanophotonic structures could address both of these issues, leading to efficiencies unattainable by planar devices with the same electronic qualities.

Light management by incorporation of randomly textured microstructures or nanostructures has been extensively investigated for crystalline silicon, amorphous silicon, and GaAs photovoltaics, 2, 7, but such approaches have not been as extensively applied to polycrystalline thin-film compound materials and devices, such as CIGS, $\mathrm{CdTe}$, and $\mathrm{Cu}_{2} \mathrm{ZnSnS}_{4}$. 8, 9, Here, we explore the underlying mechanisms of unexpectedly high absorption in random textures that were seen experimentally by the Heliovolt Corporation and compare them to other popular light trapping structures for thin-film photovoltaics. [10 12 Our simulation approach is uniquely able to extract differences between different structures by holding constituent materials parameters constant, such that performance differences are solely due to device geometry. By building on progress in other materials systems, we identify oppor- 
tunities for improvement to both the short-circuit current density $\left(J_{S C}\right)$ and open circuit voltage $\left(V_{O C}\right)$ via coupled optoelectronic simulations.

Many studies of light management in photovoltaics and solar cell architectures have focused on increased light absorption as a design objective with important implications. [13. 14] As a first approximation, this type of analysis gives a qualitative indication of how light management can increase cell efficiency. However, coupled optoelectronic modeling in which both full-field electromagnetic simulation of light absorption and scattering is combined with detailed carrier generation, transport, and recombination models results in a more complete picture. [15, 16] Accurate modeling of the spatial distribution of generated carriers is important, in addition to increasing overall absorption, because ultimately, the charge carrier collection efficiency directly affects photovoltaic efficiency. Only a full optoelectronic model can quantitatively relate optical absorption enhancement, electronic transport, and photocurrent density enhancement, and highlight electronic transport issues which can be overcome by improved optical design. For example, we observe that randomly textured CIGS absorbers can absorb the majority of the incident spectrum in a film of $700 \mathrm{~nm}$ planar equivalent thickness, but generated carriers are lost to parasitic recombination at the nearby back-contact interface. Strategies to overcome these issues are described as well other light trapping schemes for CIGS devices grown via more conventional film synthesis processes. We identify possible CIGS device architectures that enable thinning of the CIGS absorber layer to an equivalent thickness of $700 \mathrm{~nm}$ while maintaining or improving the $J_{S C}$ and/or the $V_{O C}$ compared to those of thicker planar devices.

\section{Methods}

\subsection{Optical Modelling of CIGS Absorbers}

Randomly textured CIGS thin film photovoltaic absorbers exhibit a complex film topography that results from a $\mathrm{Cu}$ and Se flux assisted re-crystallization process, leading to grain coalescence and coarsening as well as void formation via reactive mass transport. [10] These absorber layers were experimentally seen to have exceptional light trapping characteristics, motivating this numerical simulation study of the underlying light trapping mechanisms in comparison to other potential architectures. Experimental corroboration of the device performances predicted by the models herein was rendered impossible by the cessation of Heliovolt's research and development effort. However, the published experimental External Quantum Effciency (EQE) measurements qualitatively match the results herein, leading to the conclusion that our representation is a reasonable approximation of these textured devices. 10

The provided cross-sectional scanning electron microscope (SEM) images of these absorber films were used to construct models for optoelectronic simulations aimed at identifying mechanisms of light trapping. A representative cross-sectional image can be seen in Figure 17 , which is a CIGS film with equivalent planar thickness of $1.7 \mu \mathrm{m}$. This cross section was contrast-thresholded and filtered with imaging software to create a digital representation of the film. 17.

The device schematic in Figure $1 \mathrm{p}$ illustrates a simulated device structure that utilizes this absorber film morphology. The device cross section in Figure $1 \mathrm{~b}$ includes a $50 \mathrm{~nm}$ CdS conformal window layer, contacted to a CIGS absorber layer to simulate device structure resulting from conformal bath deposition. Voids which occur naturally during the recrystallization process are included in the model, and are consistent with the film morphologies observed in cross-sectional SEM micrographs. The CdS layer is coated with a $150 \mathrm{~nm}$ aluminum-doped zinc oxide (AZO) that fills the remainder of the volume and serves as a transparent front contact. The back-contact consists of a $500 \mathrm{~nm}$ molybdenum (Mo) planar thin film.

Representative devices cross sections, such as in Figure $1 \mathrm{~b}$, were rendered for full field electromagnetic simulations in two dimensions (2D) with periodic boundary conditions. 14 Electromagnetic simulations using the finitedifference time-domain (FDTD) method were performed with normally incident plane wave illumination; all optical quantities reported here were obtained as polarization averages of the transverse electric and transverse magnetic responses for the films. The absorbed fraction of the incident solar spectrum was constructed from a set of single wavelength electromagnetic simulations that were then power-weighted by the spectral irradiance of the ASTM -AM1.5G solar spectrum. The fractional gallium composition of absorbers in [10] is close to $\mathrm{x}=0.4$, and refractive index data for this composition was used to construct the complex dielectric function for CIGS absorbers in electromagnetic simulations. [18] CdS optical constants are taken from [19], ZnO from [14, and Mo from the Sopra Materials Database 20. The power absorbed in each material can be calculated from the electric field magnitude and complex refractive index at each mesh point in the simulation. The optical generation rate, $G_{o p t}$, is calculated by

$$
G_{o p t}(\lambda, \vec{r})=\frac{\epsilon^{\prime \prime}(\lambda, \vec{r})|\mathbf{E}(\lambda, \vec{r})|^{2}}{2 \hbar}
$$

where $\epsilon^{\prime \prime}$ is the imaginary part of the material's complex permittivity, $|\mathbf{E}|^{2}$ is the electric field magnitude, $\vec{r}$ is the position vector, $\lambda$ is the wavelength, and $\hbar$ is the reduced Planck's constant. [14, 15] Spatial maps of charge carrier generation are then used as inputs of the device physics simulations described in the following section. 15, 21.

\subsection{Electronic Modeling of CIGS absorbers}

The optical generation profiles for all structures depicted in Figure 11 as well for planar films were input into 
a)
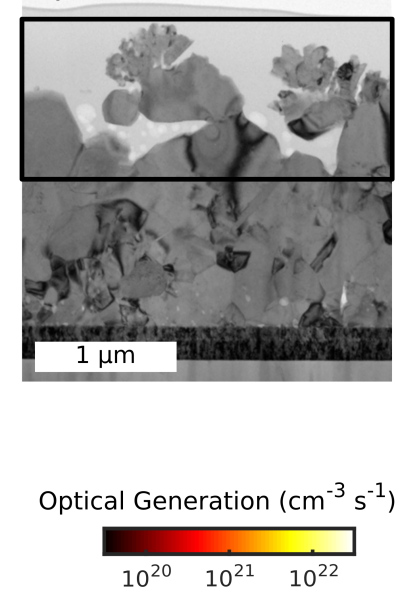

b)

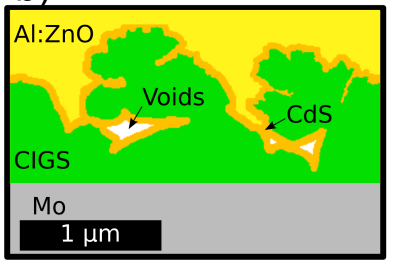

e)

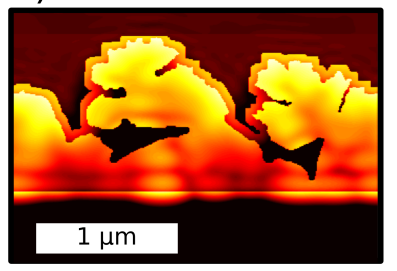

C)

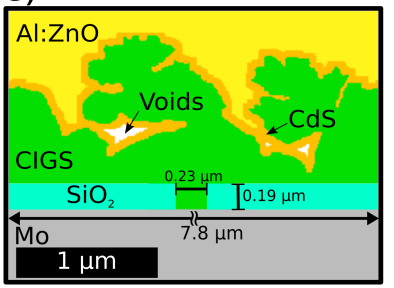

f)

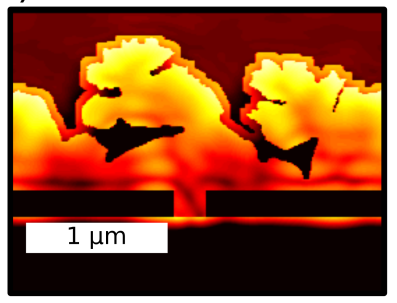

d)

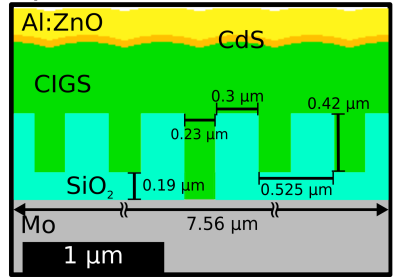

g)

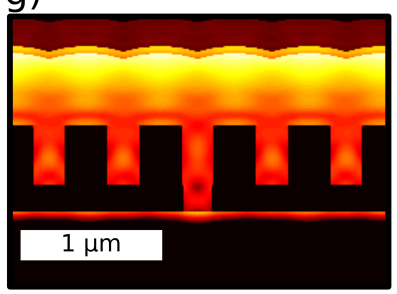

Figure 1: Simulated Cross-Sections a) A representative SEM of a focused ion beam (FIB) cross section of randomly textured CIGS absorber films is used to define the photovoltaic model for a Mo back-contact device, shown in b). In c), a $\mathrm{SiO}_{2}$ layer serves to increase reflection and define electrical line contacts, enhancing CIGS film passivation; d) shows a periodic CIGS photonic structure defined by absorber deposition on a patterned $\mathrm{SiO}_{2}$ layer with electrical line contacts. The optical generation profiles, weighted by the AM-1.5G solar spectrum, for simulated devices from b), c), and d) are shown in e), f), and g).

carrier transport simulations to numerically calculate the steady state EQE and current density-voltage (J-V) characteristics of each device. We took advantage of previously reported transport parameters for CIGS photovoltaics, 22 except where explicitly stated herein. While the reader is referred to [22] for specific materials parameters, we explicitly point out two important parameters here. First, the CIGS|Mo surface recombination velocity is set to the thermal velocity, $10^{7} \mathrm{~cm} \mathrm{~s}^{-1}$, and represents a worst-case scenario. This is in addition to a Mo|CIGS Fermi-valence band offset of $-0.2 \mathrm{eV}$ causing the valence band in the region close to the back-contact bends downward. Second, the defect density model used here more rigorously represents non-uniform lifetimes in regions with strong illumination and depletion, giving a more accurate response than the Shockley-Read-Hall (SRH) recombination model. Still, an instructive estimate of the CIGS bulk minority(majority) carrier diffusion length, as determined by the Einstein relation for diffusivity and the SRH equation of lifetime, is about $\sim 700 \mathrm{~nm}(\sim 8 \mu \mathrm{m})$ with the parameters used. Our planar device model quantitatively matches closely to Ref. [22]. The slightly lower short-circuit current densities, and correspondingly slightly lower open circuit voltages, between our model and that of Ref. [22] can be explained by the more rigorous treatment of light absorption and local photocarrier generation rate provided by FDTD simulations and the fact that the optical constants used in Ref. [22] were not reported.

This simulation method is general and can be used for any arbitrarily-shaped 2D device cross-section. Absorption in the randomly textured CIGS films was compared to planar thin films (not shown in Figure 1), CIGS films with dielectric separation layers (Figure 1c), and CIGS films deposited onto periodic dielectric structures (Figure 11d). Figures 1 and 1 d show only a subset of the larger structure periodicity defined by line contacts (the 2D approximation to point contacts), with contact periodicity of $7.8 \mu \mathrm{m}$ and $7.56 \mu \mathrm{m}$ respectively. Figure 1f and Figure 18 depict the total optical generation of those structures, respectively.

\section{Results and Discussion}

\subsection{Optical Analysis}

\subsubsection{Absorption in Self-Assembled Randomly Textured CIGS Films}

Looking at the generation map of Figure 11 immediately highlights two findings from full field electromagnetic simulation: (1) photocarrier generation hotspots in the CIGS absorber, and (2) areas of parasitic absorption in surrounding layers. Areas of high carrier generation are generally close to the randomly textured CdS/CIGS film interface. The randomly textured CIGS surface layer absorbs most of the incident light, suggesting that the underlying $1 \mu \mathrm{m}$ CIGS film beneath the surface topology in Figure 17a could be superfluous with respect to light absorption. Figure 2 a shows results for a series of randomly textured CIGS structures with varying underlying bulk film thickness, t (inset), where lighter shades of blue correspond to thinner absorber layers. Typically, in CIGS photovoltaics, absorbing layer thicknesses are on the order of 2-3 $\mu \mathrm{m}$. 23] The thickest film simulated in Figure 2a corresponds to the addition of a $1 \mu \mathrm{m}$ layer of underlying CIGS material, illustrated by the darkest blue curve, resulting in a CIGS device with a planar equivalent thickness of $1.7 \mu \mathrm{m}$, similar to the typical absorber thickness for CIGS. The 

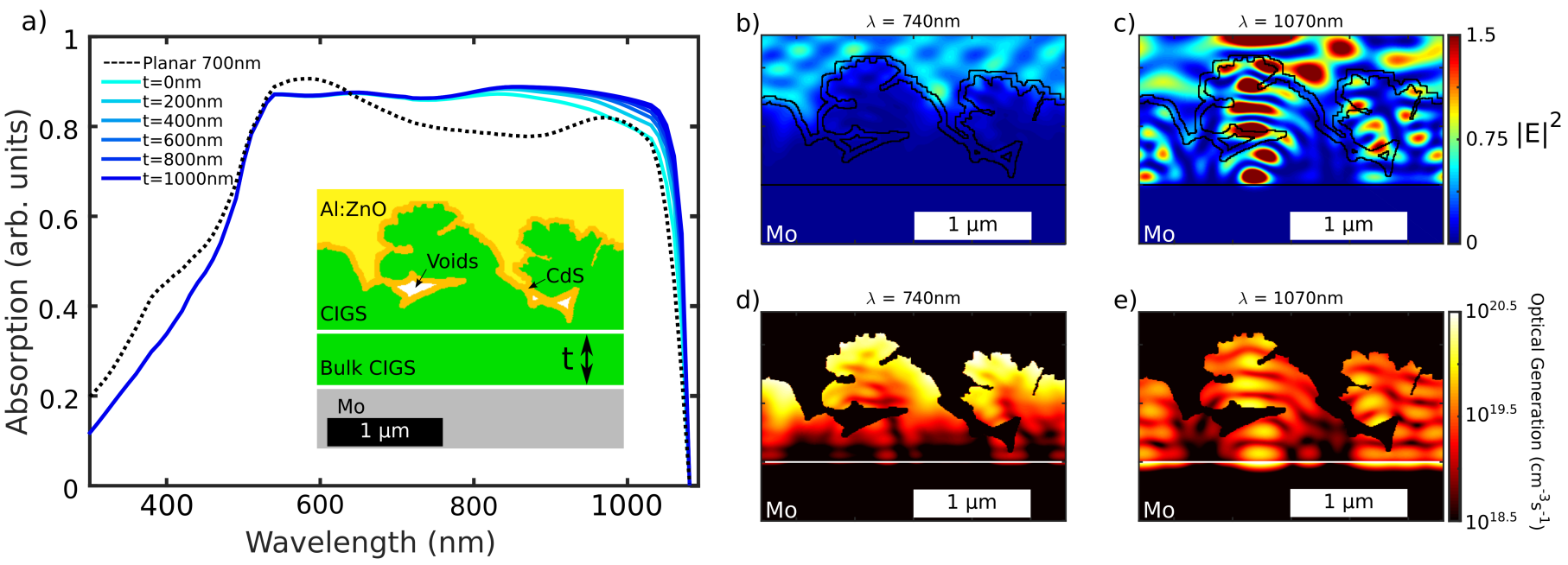

Figure 2: Randomly Textured Absorbers a) Absorption for CIGS device with a randomly textured absorber. Inset shows thickness, t, of planar CIGS film underlying randomly textured structure, which is varied between $0 \mu \mathrm{m}$ (light blue) and $1 \mu \mathrm{m}$ (dark blue). Absorption is nearly constant across the spectrum and weakly dependent on film thickness. In b) and c), magnitude of the polarization-averaged electric field intensity, $|\mathbf{E}|^{2}$, at $\lambda=740 \mathrm{~nm}$ and $\lambda=1070 \mathrm{~nm}$ respectively. Structure is outlined with black. In d) and e), optical generation rate; Mo back-contact interface indicated by horizontal white line.

high absorption of the randomly textured CIGS device is a remarkably constant across the visible and near infrared (NIR) spectrum above the CIGS bandgap, and qualitatively resembles experimentally measured EQE spectra of reference [10, as discussed later. As the underlying CIGS film thickness is varied from $1 \mu \mathrm{m}$ to $0 \mu \mathrm{m}$, the reduction in absorbed photocurrent in CIGS less than $3 \%$. This result indicates an exceptional ability of randomly textured CIGS film structures to efficiently scatter and/or absorb light that would be reflected or transmitted by a planar device with equivalent $700 \mathrm{~nm}$ CIGS thickness, represented as the black dashed curve in Figure 2 a.

Figures $2 \mathrm{~b}$ and 2 ; show TE and TM averaged electric field intensity, $|\mathbf{E}|^{2}$, profiles at $\lambda=740 \mathrm{~nm}$ and $\lambda=1070$, and Figures $2 \mathrm{~d}$ and $2 \mathrm{e}$ show the corresponding optical generation rates. These profiles indicate strong light scattering and absorption by the randomly textured CIGS films. Absorption in the planar device begins to decline at wavelengths beyond $600 \mathrm{~nm}$ due to increasing reflection, while absorption in the randomly textured CIGS absorbers does not decline until $\sim 740 \mathrm{~nm}$ due to the longer optical path lengths and scattering promoted by the texture, as demonstrated in Figures $2 \mathrm{~b}$ and $2 \mathrm{~d}$. Beyond $740 \mathrm{~nm}$, the randomly textured CIGS begins to transmit light, and a nonzero thickness for the underlying CIGS layer is required for full absorption. In regions of the spectrum where light reaches the Mo back-contact, it is parasitically dissipated in the metal. The significant absorption loss in the Mo is due to its refractive index being relatively well-matched to CIGS, leading to a low reflectivity interface. 8, 24] Typically, the metal back-contact in a solar cell should serve as a good back reflector to enable multi-pass absorption, in addition to its role as an electrical contact. However, in a CIGS solar cell, Mo is typically used to create a small to zero Schottky contact to CIGS and for its $\mathrm{Na}^{+}$perme- ability, despite its undesirable optical properties. 25, 26]

Furthermore, Figures 2r and 22 indicate field intensity enhancement in the lobes of the CIGS randomly textured structure; these lobes are characterized by their location above a void. The sharp refractive index contrast at the CIGS $|\mathrm{CdS}|$ void interface results in reflection back into the absorber material. Thus these lobes effectively act as monolithically integrated low $\mathrm{Q}$ resonators. These lobes are also areas of high electric potential, implying that a large fraction of the carriers generated in these regions are collected.

Figure 3 a shows the spectral absorption of each material in the randomly textured CIGS structure with no underlying planar CIGS film (Figure $1 \mathrm{~b}, 1$ ) to aid the identification of opportunities for more efficient light management. The solid black curve overlaid onto the absorption spectra represents the simulated EQE, with the difference between absorption and EQE indicating device Internal QE. The most significant fraction of absorption occurs in the CIGS layer (blue). However, at wavelengths shorter than the AZO and CdS bandgaps, CIGS absorption is significantly diminished due to parasitic interband transitions in the CdS and AZO layers. The parasitic absorption at shorter wavelengths accounts for $\sim 11 \%$ of the total incident photons above the CIGS bandgap $\left(4.4 \mathrm{~mA} \mathrm{~cm}^{-2}\right)$. We note that AZO parasitic absorption here is greater in the randomly textured structure than in a traditional planar design because of the increased total AZO material required to make a conformal front contact here. While light absorbed in the AZO is completely lost, the simulated EQE curve (solid black) shows that some carriers generated in the $\mathrm{CdS}$ window layer do contribute to the short circuit current. However, this collection from the CdS is inefficient due to the high defect concentration in the window layer. 22] Optically, a thin AZO layer would 
be ideal, but electronically, a thin AZO layer would incur increased resistive losses. Similarly, further thinning of the CdS could also be detrimental, causing pin holes and shunts among other transport-related issues. [23, 27] Use of wider band gap heterojunction window layer materials is a potential alternative to minimize photocurrent lost in $\mathrm{CdS}$, and is currently under investigation. [26, 28,

Figure $3 \mathrm{~b}$ shows the material parsed absorption for a textured CIGS device with $\mathrm{t}=1 \mu \mathrm{m}$. The absorption response in the blue wavelengths is the same because the device is not optically thin. Unsurprisingly, the major difference between the thick device and the thin device is a reduced portion of NIR spectrum is absorbed by the Mo back-contact. The solid black curve overlay represents the simulated EQE of the thicker device, which has increased proportional to the reduced absorption in the Mo backcontact in the NIR. Additionally, the dashed black curves show experimental data from Ref. 10 for comparison. Optically, the 1-reflection (1-R) curve shows that the overall absorption is closely matched. More importantly, the experimental and simulated EQE curves display a reasonably similar behavior. Both EQE curves show a large, flat response across the solar spectrum into the NIR enabled by the strong scattering of longer wavelengths. The difference in the higher simulated EQE curve can be ascribed to using literature values for electronic properties of the material, but the agreement enables us to draw conclusions about the factors that are most important in structured devices.

Thus as shown in Figure $3 a$ and b, the other optical loss mechanisms are reflection at the front surface interface and absorption by the Mo back-contact. Front surface reflection losses can be broadly addressed by applying at typical $\mathrm{MgF}_{2}$ anti-reflection coating (see SI1). The loss in the Mo can be mitigated by appropriate design of dielectric reflection layers to give high refractive index contrast along with promising electronic transport behavior, [12] as discussed below.

\subsubsection{Thin Film and Patterned Dielectric Layers}

Parasitic losses in the Mo are difficult to avoid for submicrometer absorber layer thicknesses. However, these losses can be reduced by increasing reflectance at the CIGS |Mo interface. A dielectric separation layer with Mo line contacts, as shown in Figures 1e and 1f, has been proposed to increase reflectance at this interface. [12, 29, 30 To maximize the reflectance of this new dielectric interface, the dielectric layer should have a low index and its thickness should be designed for destructive interference at wavelengths near the band edge. As an example, low refractive index silica sol gel $(n=1.42)$ layers could be utilized and patterned via nanoimprint lithography. 31, 32, Due to the nonplanar film morphology studied here, we optimized this low index layer for absorbed photocurrent using FDTD and partial spectral averaging for all device geometries. This approach more closely resembles experimental results due to the small, random thickness vari- a)

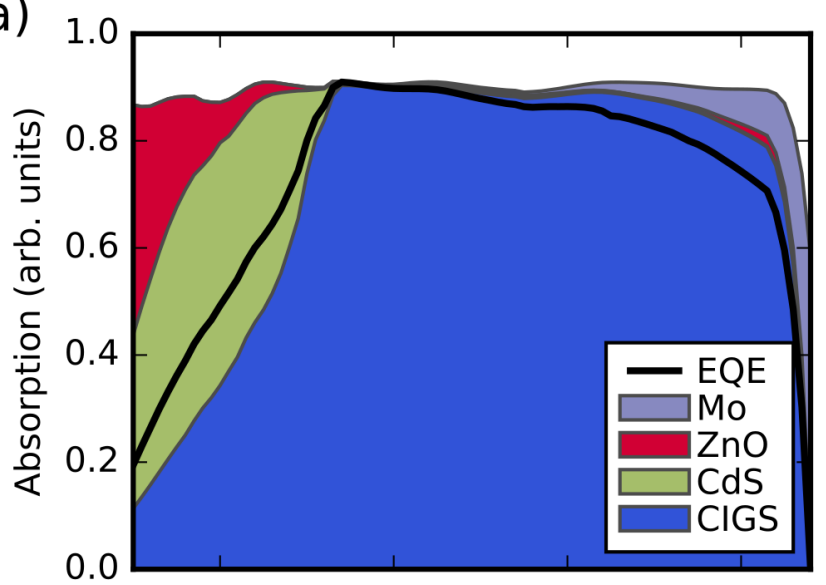

b)

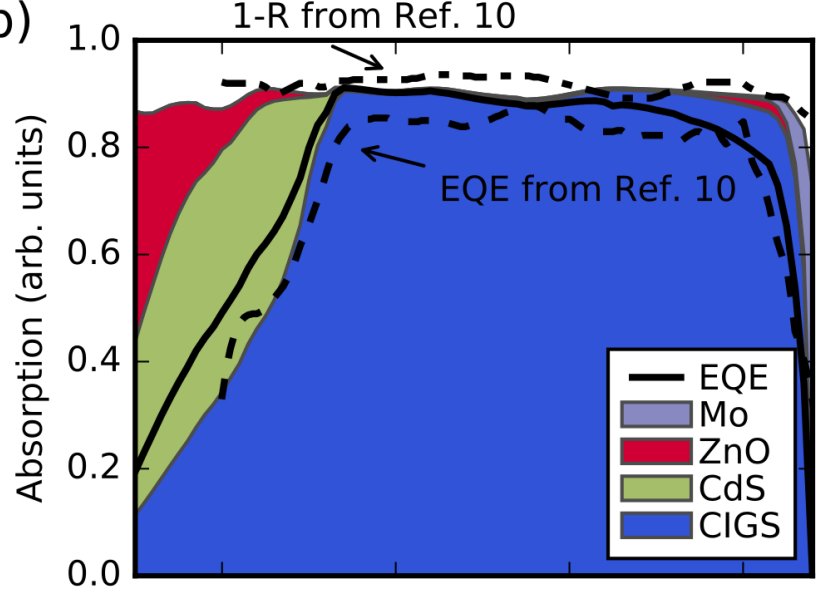

C)

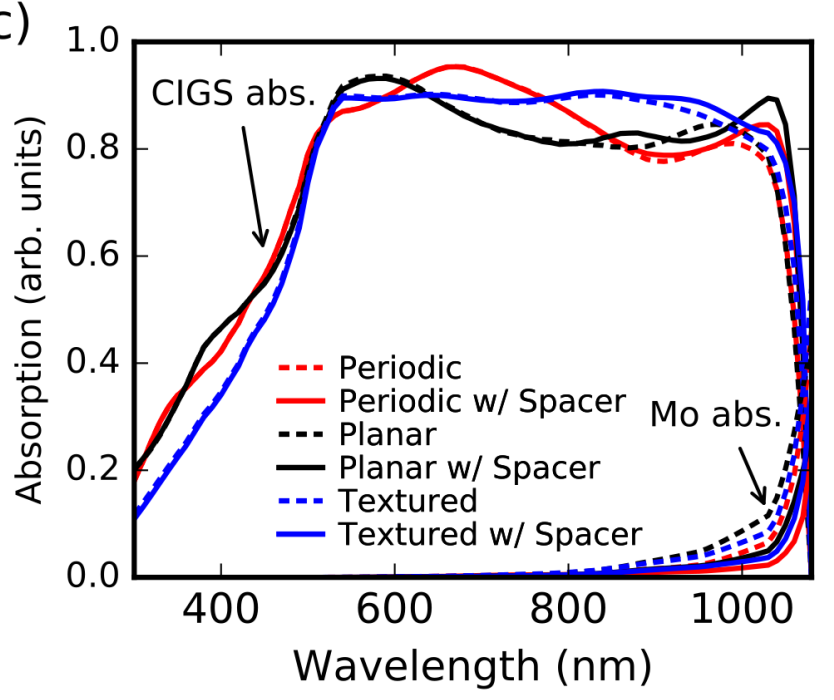

Figure 3: Absorption in Component Materials a) Materials parsed absorption in a simulated randomly textured device with a planar equivalent of $700 \mathrm{~nm}, \mathrm{t}=0 \mathrm{~nm}$. The black curve overlaid on the plot is the simulated device external quantum efficiency. b) The same as in a, but for $\mathrm{t}=1000 \mathrm{~nm}$. The $1-\mathrm{R}$ and experimental EQE from 10 are provided in dashed lines for direct comparison to the model here. In c), comparison of CIGS and Mo absorption for different device structures with and without dielectric layers between the absorber and back-contact. 
ations of real films. 33 An optimum layer thickness of $\sim 190 \mathrm{~nm}$ was found. This low refractive index layer thickness maximizes its reflectance at $\lambda=1040 \mathrm{~nm}$, seen as a CIGS absorption peak in Figure 3 .

Figure 3 . demonstrates the reduction of parasitic Mo absorption and correspondingly increased CIGS absorption due to enhanced CIGS back interface reflectance for the structures depicted in Figure 1 as well as a planar thin film. First, comparing the dashed black curve and the solid black curve, we see that insertion of the low index layer into the planar structure increases absorption in a planar film slightly at wavelengths where the CIGS is optically thin, beginning around $800 \mathrm{~nm}$. The largest boost in absorption is near the band edge, where the dielectric layer is designed to reflect light back into the CIGS absorber via destructive interference.

Alternatively, periodically ordered structures, such as an array of pillars, can also improve absorption in the CIGS layer (Figure $1 \mathrm{~d}, 1 \mathrm{~g}$ ). An ordered structure, that has again been optimized for absorbed photocurrent, with a period of $525 \mathrm{~nm}$, a height of $420 \mathrm{~nm}$, and feature width of $300 \mathrm{~nm}$ imparts periodic structuring to a conformably deposited CIGS absorber layer. This strongly increases CIGS absorption between $600 \mathrm{~nm}$ and $800 \mathrm{~nm}$, seen in the red dashed curve of Figure 3 , corresponding to the maximum of solar photon flux. This periodic structure has similar anti-reflection properties as the randomly textured absorbers, where the conformal and periodic surface texture preferentially scatters light into the absorber at these wavelengths, seen as the generation by the junction in Figure 13. 34, 35] Unlike the randomly textured absorber, a periodic structure can be controllably reproduced via methods such as nanoimprint lithography. Augmenting the periodically ordered structures with a $190 \mathrm{~nm}$ dielectric layer at the Mo interface gives an additional absorption increase close to the band-edge, exhibited by the solid red curve of Figure 3 . The overall absorption spectrum closely resembles a superposition of the effects from the two light trapping mechanisms, suggesting multiple strategies can be combined to achieve overall broadband enhancement. $\mathrm{A}^{1 / 4}$ wavelength $\mathrm{MgF}_{2}$ anti-reflection coating tuned to $\lambda \sim 900 \mathrm{~nm}$ is discussed in Supplementary Information section SI1.

Combining a dielectric reflection layer with randomly textured CIGS absorbers does not result in the same absorption enhancement as with the lithographically patterned and planar films, as seen when comparing the solid and dashed blue curves of Figure 3. The reduced enhancement is attributed to the strong scattering of incident light, which inhibits the destructive interference condition within the dielectric layer. Nevertheless, a modest broadband increase in absorption is seen at longer wavelengths where the CIGS absorber is optically thin, beginning around $\lambda=900 \mathrm{~nm}$. This corresponds to the fraction of light that is reflected back into the absorber at the CIGS $\mid \mathrm{SiO}_{2}$ interface, and can be inferred by comparing the Mo absorption curves of the three architectures with- out and with dielectric separation layers (dashed versus solid) in Figure 3c. The dielectric layer clearly decreases the parasitic absorption near the band edge in all cases, verifying the enhanced back interface reflectance.

\subsection{Carrier Transport Analysis}

\subsubsection{Randomly Textured Absorbers}

The absorbed photocurrent can be determined for optical structures with improved light management, such as the randomly textured CIGS absorbers described here, but the corresponding device transport and electrical performance cannot be predicted from optical models alone. Common assumptions in existing literature on optical modeling of photocurrent enhancement include unity or constant internal quantum efficiency, neglecting wavelength and/or spatially dependent collection probabilities. $36-$ 39] In some systems this assumption is well justified, [14, 40] while, in others, a fully coupled optoelectronic model is necessary for qualitatively and quantitatively accurate results. [16, 21] Here, a fully combined optical simulation and carrier transport analysis (c.f., EQE curves in Figure $3 a, b)$ indicates that photons and electrons are more efficiently collected at shorter wavelengths than at longer wavelengths in the randomly textured device. All generated carriers are collected near $550 \mathrm{~nm}$, while only about $80 \%$ of generated carriers are collected at $1040 \mathrm{~nm}$, given by the ratio of the absorbed photons in the CIGS and the EQE curve. Minority electrons generated near the CIGS|Mo interface are swept to the back-contact and recombine. This only occurs at longer wavelengths with correspondingly low absorption coefficients of the CIGS absorber.

The illuminated J-V curves for various underlying CIGS film thickness are shown in Figure 4. The change in $J_{S C}$ between the $\mathrm{t}=700 \mathrm{~nm}$ and $\mathrm{t}=1700 \mathrm{~nm}$ devices is $0.8 \mathrm{~mA} \mathrm{~cm}^{-2}$, and this increase is less than $3 \%$ as previously predicted by the optical analysis. In contrast the $V_{O C}$ increases significantly, from $605.3 \mathrm{mV}$ to a maximal value of $634.2 \mathrm{mV}$. The small increase in $J_{S C}$ alone cannot account for the substantial increase of the $V_{O C}$. The observed $V_{O C}$ gain is instead attributed to the spatial separation of carrier generation in thicker films from parasitic recombination at the back-contact, giving minority carriers generated at depth a greater chance of diffusing to the space charge region near the diode junction. 1] The estimated bulk minority carrier diffusion length is $\sim 700 \mathrm{~nm}$, and for sufficiently thick CIGS films, the majority of photogenerated carrier are at least this distance from the back-contact, so recombination is reduced and the $V_{O C}$ approaches a maximal value.

Here, the $V_{O C}$ of a randomly textured device with $1 \mu \mathrm{m}$ of underlying CIGS shows a slightly diminished VOC compared to a planar device of the equivalent $1.7 \mu \mathrm{m}$ of material $(634.2 \mathrm{mV}$ and $637.8 \mathrm{mV}$ respectively). This can be expected due to the increasing junction area, and therefore dark recombination. However, these simulations found the 


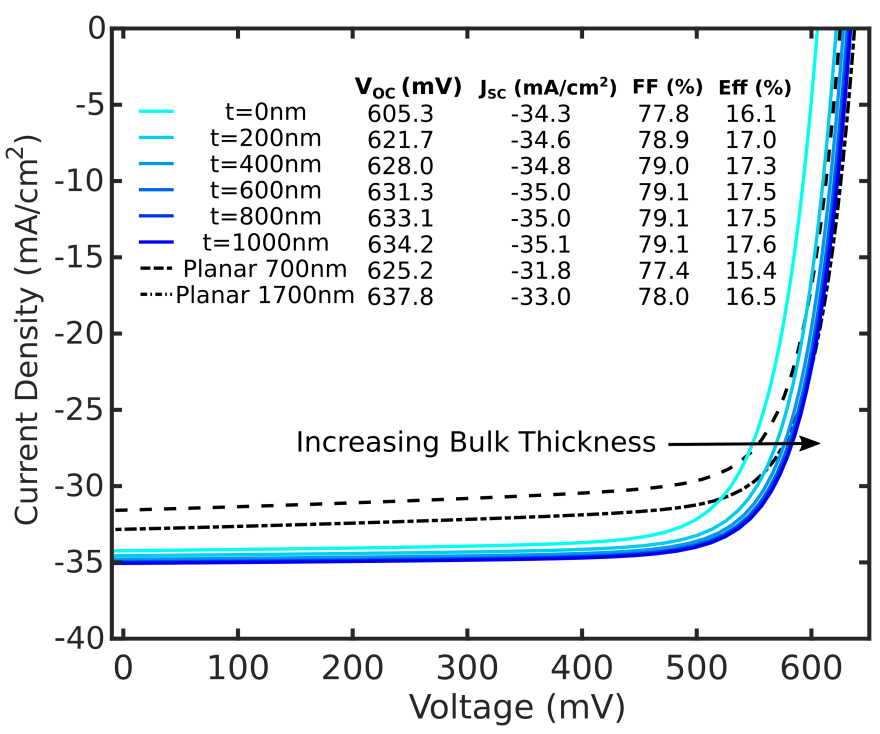

Figure 4: Randomly Textured CIGS Photovoltaic Response Illuminated simulations of devices with varying CIGS bulk thickness, t, (c.f., inset of Figure 2a) compared with the simulated J-V curves of equivalent planar devices. Extracted J-V parameters are given by inset.

increase in current overcomes the loss in voltage when considering the overall efficiency, provided back-surface recombination is mitigated by sufficient underlying thickness. For these textured devices it is equally important that the surface recombination velocity (SRV) at the $\mathrm{CdS} \mid \mathrm{CIGS}$ interface is kept below $10^{3} \mathrm{~cm} \mathrm{~s}^{-1}$ or the substantially increased interface area will quickly reduce device fill factor as the SRV at this interface increases (See SI2). These results encourage further investigation of this system into other potential limiting issues, including increased shunting, potentially high defect concentrations at sharp features, and this additional need to control the junction interface recombination.

\subsubsection{Electronic Benefits of Thin Film Dielectric Layers}

Thin film dielectric separation layers embedded between the CIGS absorber and Mo contact provide a barrier to minority carrier recombination if appropriate surface passivation can be achieved at CIGS|dielectric interfaces. SRVs in the range of $10^{2}-10^{4} \mathrm{~cm} \mathrm{~s}^{-1}$ have been reported for alumina dielectric layers, and similar values could be expected with other oxides such as silica. 9, 30 Figure 5 shows light $\mathrm{J}-\mathrm{V}$ parameters of the textured device in Figure 1. while parametrically varying the SRV at the CIGS|dielectric separation layer interfaces. The $V_{O C}$ remains large until $\sim 10^{3} \mathrm{cms}^{-1}$ and then drops monotonically for larger surface recombination velocities; similar results are seen for the $J_{S C}$ and efficiency. The periodically structured device suffers the most in terms of $J_{S C}$ and fill factor with this increase in SRV due to its larger interface area. The planar device maintains the most stable $V_{O C}$, but loses $J_{S C}$ must faster than the randomly textured device. If low SRVs can be achieved, a line/point
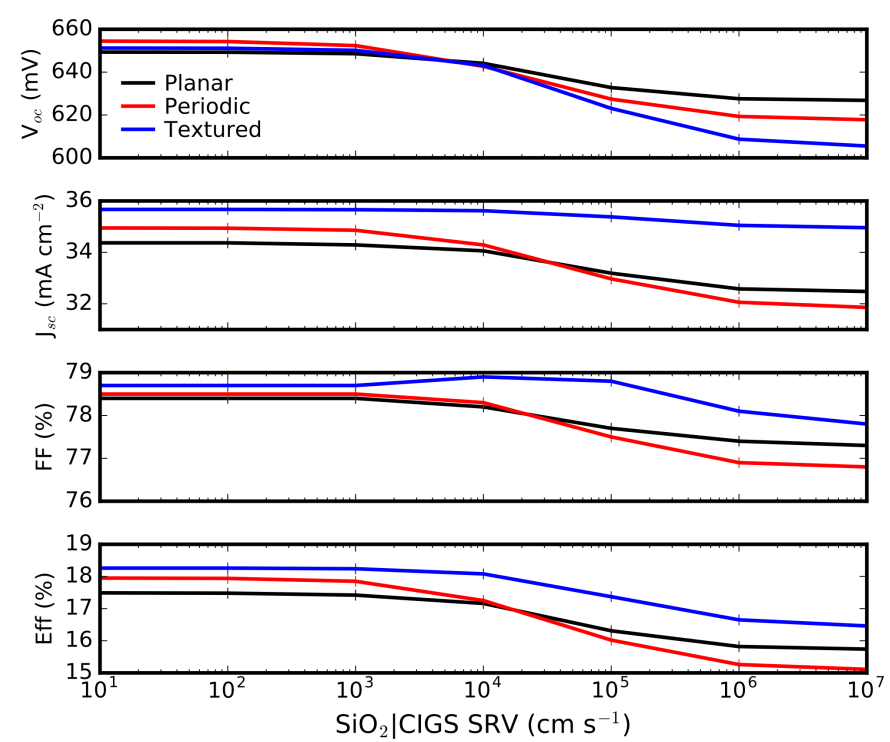

Figure 5: Effect of Dielectric Surface Recombination $V_{O C}$, $J_{S C}$, fill factor, and efficiency of simulated devices with dielectric layers $(\mathrm{t}=0 \mathrm{~nm}$ for the randomly textured device) that define line contacts for parametrically varied CIGS|SiO 2 interface surface recombination velocity.

contact scheme with a dielectric reflection layer would represent an attractive device design to increase both the $J_{S C}$ and $V_{O C}$ of any thin-film device architecture. [9, 30] The improvements when adding the dielectric layer are summarized in Table 11. along with the device performance when adding a $\mathrm{MgF}_{2}$ ARC that has been optimized for photocurrent absorbed by the CIGS (SI1).

The back-contact passivation by dielectric separation layers in sub-micron thickness CIGS films is only required when a band offset exists between the CIGS and the Mo contact. A thin layer of $\mathrm{MoSe}_{2}$ is sometimes used to create an indirect ohmic contact to Mo, but this has been reported to cause delamination issues in cases when this layer is excessively thick. 26] Ga grading has also been proposed to avoid minority carrier loss to the back-contact. [1] We examined the sensitivity of the randomly textured CIGS device to this offset in the Supplementary Information (SI3) and find that, electronically, adding dielectric reflection layers have a similar effect as making this offset more positive, but, optically, dielectric layers give more $J_{S C}$ due to increased double pass absorption. Thus, sparsely arrayed line contacts are the preferred design to simultaneously minimize surface recombination and enable the required majority carrier transport while increasing double pass absorption.

Due to computational limitations, we were unable to simulate line contacts with spacing of greater than $7.8 \mu \mathrm{m}$. Thus the ultimate potential of the dielectric layer line contact design was not fully optimized in this study. We note that majority carriers are collected at the back-contact in this scheme, so the period of the line contacts can be on the order of the majority carrier diffusion length, as is the case here. The line contacts were $230 \mathrm{~nm}$ in width for all 
Table 1: Summary of JV Parameters for Different Device Configurations

\begin{tabular}{|c|c|c|c|c|}
\hline Simulated Device & $V_{O C}(\mathrm{mV})$ & $J_{S C}\left(\mathrm{~mA} \mathrm{~cm}^{-2}\right)$ & $\mathrm{FF}(\%)$ & Effciency $(\%)$ \\
\hline Equivalent Thickness Planar & 625.2 & 31.8 & 77.4 & 15.4 \\
\hline Periodic Structure $^{1}$ & 641.0 & 33.6 & 78.5 & 16.9 \\
\hline Randomly Textured & 605.3 & 34.3 & 77.9 & 16.1 \\
\hline \multicolumn{5}{|c|}{ Simulated Device with $190 \mathrm{~nm}$ Dielectric Layer ${ }^{1}$} \\
\hline Equivalent Thickness Planar & 648.6 & 34.3 & 78.4 & 17.4 \\
\hline Periodic Structure & 652.4 & 34.9 & 78.5 & 17.9 \\
\hline Randomly Textured & 654 & 35.2 & 78.4 & 18.1 \\
\hline \multicolumn{5}{|c|}{ Simulated Device with $190 \mathrm{~nm}$ Dielectric Layer and $\mathrm{ARC}^{1}$} \\
\hline Equivalent Thickness Planar & 653.1 & 37.4 & 78.4 & 19.1 \\
\hline Periodic Structure $^{2}$ & 654.8 & 37.6 & 78.5 & 19.3 \\
\hline Randomly Textured & 655.8 & 38.3 & 78.5 & 19.7 \\
\hline
\end{tabular}

1 The SRV at the CIGS $\mid \mathrm{SiO}_{2}$ interface is $10^{3} \mathrm{~cm} \mathrm{~s}^{-1}$

2 This ARC was $166 \mathrm{~nm}$ instead of $110 \mathrm{~nm}$

devices as in Figure 17, d. We observed that decreasing the contact opening could further enhance the passivation effect, indicating that recombination is not negligible even for this line contact spacing of $7.8 \mu \mathrm{m}$. However, very small line contacts on the order of $150 \mathrm{~nm}$ began to limit the $J_{S C}$. Additionally, experimentally contacting the Mo may be difficult with excessively small vias. We also recognize the beneficial role of $\mathrm{Na}^{+}$ion diffusion through the Mo back-contact should not be ignored, but incorporation of sodium fluoride into dielectric reflection layer has been reported before. [12, 29, 30] It is proposed first here that direct incorporation of $\mathrm{Na}^{+}$into a sol-gel layer used in nanoimprint fabrication could be a scalable solution to controlled $\mathrm{Na}^{+}$incorporation.

\section{Conclusion}

We report coupled optoelectronic simulations for light trapping and surface passivation in CIGS photovoltaics, and indicate designs to match or exceed the performance of thick planar absorbers. For randomly textured CIGS cells, the majority of light absorption occurs in the textured region near the junction. Long wavelength light is effectively scattered giving a flat, broadband absorption spectrum. Other light trapping strategies were examined, including dielectric separation layers located between the CIGS absorber and Mo back-contact. These layers can be designed to preferentially reflect near band-gap light back into the absorber to reduce parasitic Mo absorption. Periodic dielectric structures can also give enhancement by increasing scattering into the device. Employing various combinations of different light trapping strategies in these thin film devices demonstrates synergistic effects. For example, combining dielectric layers and periodic nanostructures gives absorption spectra characterized by superposition of these two different effects.

These optoelectronic models also addressed the other main challenge to sub-micron absorbers, namely, minority carrier recombination at the back-contact. Dielectric separation layers show the dual promises of surface passivation while enhancing double-pass absorption. Finally, we compared each device with the addition of a $1 / 4$ wavelength $\mathrm{MgF}_{2}$ anti-reflection coating and found that a randomly textured absorber with $\mathrm{t}=0 \mathrm{~nm}(700 \mathrm{~nm}$ planar equivalent) with a $190 \mathrm{~nm}$ dielectric separation layer shows the largest potential efficiency (see SI1). These efficiencies represent an upper bound to the CIGS architectures simulated here. Experimentally, planar devices may be easier to achieve due to the less stringent requirements on SRV of the CIGS $\mid \mathrm{SiO}_{2}$ and CIGS $\mid \mathrm{CdS}$ interfaces. However, randomly or periodically structured CIGS devices with reasonably achievable CIGS $\mid \mathrm{SiO}_{2} \mathrm{SRV}$ values significantly out-performed the planar architectures. Devices utilizing this design approach could result in improved CIGS photovoltaic efficiency with a significant reduction in absorber layer thickness, and corresponding reduction in material consumption and cost.

\section{Acknowledgments}

The authors thank Hal Emmer, Chris T. Chen, Yulia Tolstova, and Stefan Olmelcheko for helpful discussions. Dr. Stanbery acknowledges the HelioVolt team of co-inventors that developed the processing technology to create these nanotextured absorbers. [10] This work was supported by the U.S. Department of Energy and the Bay Area Photovoltaic Consortium under award number DEEE0004946 (C.R.B. and D.M.C.) and the Joint Center for Artificial Photosynthesis (KT.F. and H.A.A.), a DOE Energy Innovation Hub, supported through the Office of Science of the U.S. Department of Energy under Award No. DE-SC0004993. K. T. Fountaine was supported by the National Science Foundation Graduate Research Fellowship under Grant No DGE-1144469. 


\section{References}

[1] M. Gloeckler, J. R. Sites, Potential of submicrometer thickness $\mathrm{Cu}(\mathrm{In}, \mathrm{Ga}) \mathrm{Se} 2$ solar cells Journal of Applied Physics 98 (10) (2005) 103703. doi:10.1063/1.2128054

URL http://link.aip.org/link/JAPIAU/v98/i10/p103703/ s1\{\&\}Agg=doihttp://scitation.aip.org/content/aip/ journal/jap/98/10/10.1063/1.2128054

[2] M. D. Kelzenberg, S. W. Boettcher, J. a. Petykiewicz, D. B. Turner-Evans, M. C. Putnam, E. L. Warren, J. M. Spurgeon, R. M. Briggs, N. S. Lewis, H. a. Atwater, Enhanced absorption and carrier collection in $\mathrm{Si}$ wire arrays for photovoltaic applications Nature Materials 9 (4) (2010) 368-368. doi:10.1038/nmat2727

URL http://dx.doi.org/10.1038/nmat2635http://www . nature.com/doifinder/10.1038/nmat2727

[3] P. Campbell, M. a. Green, Light trapping properties of pyramidally textured surfaces Journal of Applied Physics 62 (1) (1987) 243. doi:10.1063/1.339189

URL http://scitation.aip.org/content/aip/journal/jap/ 62/1/10.1063/1.339189

[4] E. Yablonovitch, Statistical ray optics JOSA 72 (7) (1982) 899. doi:10.1364/JOSA.72.000899

URL http://www.opticsinfobase.org/abstract.cfm?URI= josa-72-7-899http://www.opticsinfobase.org/abstract. cfm?uri=josa-72-7-899

[5] H. W. Deckman, C. R. Wronski, H. Witzke, E. Yablonovitch, Optically enhanced amorphous silicon solar cells, Applied Physics Letters 42 (11) (1983) 968-970. doi:10.1063/1.93817

[6] J. Müller, B. Rech, J. Springer, M. Vanecek, TCO and light trapping in silicon thin film solar cells, Solar Energy 77 (6) (2004) 917-930. doi:10.1016/j.solener.2004.03.015 URL http://linkinghub.elsevier.com/retrieve/pii/ S0038092X04000647

[7] J. Grandidier, R. a. Weitekamp, M. G. Deceglie, D. M. Callahan, C. Battaglia, C. R. Bukowsky, C. Ballif, R. H. Grubbs, H. a. Atwater, Solar cell efficiency enhancement via light trapping in printable resonant dielectric nanosphere arrays, Physica Status Solidi (a) 210 (2) (2013) 255-260. doi:10.1002/pssa. 201228690

URL http://doi.wiley.com/10.1002/pssa.201228690

[8] C. Colin, I. Massiot, A. Cattoni, N. Vandamme, C. Dupuis, N. Bardou, I. Gerard, N. Naghavi, J.-F. Guillemoles, J.-L. Pelouard, S. Collin, Broadband light-trapping in ultra-thin nano-structured solar cells 2013, p. 86200C. doi: $10.1117 / 12.2004269$

URL http://proceedings.spiedigitallibrary.org/ proceeding. aspx?doi=10.1117/12.2004269

[9] C. van Lare, G. Yin, A. Polman, M. Schmid, Light Coupling and Trapping in Ultrathin $\mathrm{Cu}(\mathrm{In}, \mathrm{Ga}) \mathrm{Se} 2$ Solar Cells Using Dielectric Scattering Patterns, ACS Nanddoi:10.1021/acsnano. $5 \mathrm{~b} 04091$

URL http://pubs . acs . org/doi/10.1021/acsnano .5b04091

[10] B. Sang, D. Lu, R. M. Miller, C. R. Martinez, M. Kim, S.-S. Moon, B. J. Stanbery, Nanostructured CIGS Absorber Surface for Enhanced Light Trapping (2014).

URL

https://patentscope.wipo.int/search/en/ W02014028542

[11] M.-C. van Lare, A. Polman, Optimized Scattering Power Spectral Density of Photovoltaic Light-Trapping Patterns, ACS Photonics (2015) 150626105810001doi:10.1021/ph500449v URL http://pubs .acs .org/doi/abs/10.1021/ph500449v

[12] B. Vermang, J. T. Wätjen, V. Fjällström, F. Rostvall, M. Edoff, R. Gunnarsson, I. Pilch, U. Helmersson, R. Kotipalli, F. Henry, D. Flandre, Highly reflective rear surface passivation design for ultra-thin $\mathrm{Cu}(\mathrm{In}, \mathrm{Ga}) \mathrm{Se} 2$ solar cells Thin Solid Films 582 (2015) 300-303. doi:10.1016/j.tsf.2014.10.050

URL http://linkinghub.elsevier.com/retrieve/pii/ S0040609014010116

[13] D. Callahan, K. Horowitz, H. Atwater, Light trapping in ultrathin silicon photonic crystal superlattices with randomlytextured dielectric incouplers, Optics Express 21 (25) (2013)
4239-4245. doi:10.1364/0E.21.030315

URL http://www.opticsinfobase.org/oe/fulltext.cfm?uri= oe-21-25-30315

[14] V. E. Ferry, A. Polman, H. a. Atwater, Modeling Light Trapping in Nanostructured Solar Cells, ACS Nano 5 (12) (2011) 1005510064. doi:10.1021/nn203906t URL http://www.ncbi.nlm.nih.gov/pubmed/22082201http:// pubs.acs.org/doi/abs/10.1021/nn203906t

[15] M. G. Deceglie, V. E. Ferry, A. P. Alivisatos, H. a. Atwater, Design of Nanostructured Solar Cells Using Coupled Optical and Electrical Modeling Nano Letters 12 (6) (2012) 2894-2900. doi:10.1021/nl300483y

URL http://www.ncbi.nlm.nih.gov/pubmed/22574816http:// pubs.acs.org/doi/abs/10.1021/nl300483y

[16] X. Li, N. P. Hylton, V. Giannini, K.-H. Lee, N. J. Ekins-Daukes, S. a. Maier, Bridging electromagnetic and carrier transport calculations for three-dimensional modelling of plasmonic solar cells., Optics express 19 Suppl 4 (May) (2011) A888-A896. doi:10.1364/OE.19.00A888

[17] M. D. Abràmoff, P. J. Magalhães, S. J. Ram, Image processing with imageJ (2004). doi:10.1117/1.3589100.

URL http://biomedicaloptics.spiedigitallibrary.org/ article.aspx?doi=10.1117/1.3589100

[18] M. Alonso, M. Garriga, C. Durante Rincón, E. Hernández, M. León, Optical functions of chalcopyrite CuGa x In 1-x Se 2 alloys Applied Physics A: Materials Science \& Processing 74 (5) (2002) 659-664. doi:10.1007/s003390100931 URL http://link. springer.com/10.1007/s003390100931

[19] R. E. Treharne, a. Seymour-Pierce, K. Durose, K. Hutchings, S. Roncallo, D. Lane, Optical Design and Fabrication of Fully Sputtered CdTe/CdS Solar Cells, Journal of Physics: Conference Series 286 (2011) 012038. doi :10.1088/1742-6596/286/1/ 012038

URL http://stacks.iop.org/1742-6596/286/i=1/a=012038? $k e y=c r o s s r e f . f 72 f d a 0 f c f 5 a a 2 c d 75968988273 f 5 c e 4$

[20] Sopra Materials Database URL

http://www.filmetrics.com/ refractive-index-database/Mo/Molybdenum

[21] K. T. Fountaine, H. a. Atwater, Mesoscale modeling of photoelectrochemical devices: light absorption and carrier collection in monolithic, tandem, Si-WO_3 microwires Optics Express 22 (S6) (2014) A1453. doi:10.1364/OE.22.0A1453 URL http://www.opticsinfobase.org/abstract.cfm? URI=oe-22-S6-A1453https://www .osapublishing.org/oe/ abstract.cfm?uri=oe-22-S6-A1453

[22] M. Gloeckler, A. L. Fahrenbruch, J. R. Sites, Numerical modeling of CIGS and CdTe solar cells: setting the baseline, in: Proceedings of 3rd World Conference onPhotovoltaic Energy Conversion, 2003.

[23] I. Repins, S. Glynn, J. Duenow, T. J. Coutts, W. K. Metzger, M. A. Contreras, Required Material Properties for High-Efficiency CIGS Modules, no. July, 2009, pp. 74090M$74090 \mathrm{M}-14$. doi:10.1117/12.828365.

URL http://proceedings.spiedigitallibrary.org/ proceeding. aspx?articleid $=1340399$

[24] N. Dahan, Z. Jehl, T. Hildebrandt, J.-J. Greffet, J.-F. Guillemoles, D. Lincot, N. Naghavi, Optical approaches to improve the photocurrent generation in $\mathrm{Cu}(\mathrm{In}, \mathrm{Ga}) \mathrm{Se} 2$ solar cells with absorber thicknesses down to $0.5 \mu \mathrm{m}$ Journal of Applied Physics 112 (9) (2012) 094902. doi:10.1063/1.4762004

URL http://scitation.aip.org/content/aip/journal/jap/ 112/9/10.1063/1.4762004

[25] K. Orgassa, H. Schock, J. Werner, Alternative back contact materials for thin film $\mathrm{Cu}(\mathrm{In}, \mathrm{Ga}) \mathrm{Se} 2$ solar \begin{tabular}{l|lllll}
\hline cells & Thin Solid Films $431-432$ & (03) & (2003) & 387-391.
\end{tabular} doi:10.1016/S0040-6090(03)00257-8

URL http://linkinghub.elsevier.com/retrieve/pii/ S0040609003002578

[26] B. J. Stanbery, Copper Indium Selenides and Related Materials for Photovoltaic Devices, Critical Reviews in Solid State and Materials Sciences 27 (2) (2002) 73-117. 
doi: 10.1080/20014091104215

URL http://www.tandfonline.com/doi/abs/10.1080/ 20014091104215

[27] M. a. Contreras, M. J. Romero, B. To, F. Hasoon, R. Noufi, S. Ward, K. Ramanathan, Optimization of CBD CdS process in high-efficiency $\mathrm{Cu}(\mathrm{In}, \mathrm{Ga}) \mathrm{Se} 2$-based solar cells, in: Thin Solid Films, Vol. 403-404, 2002, pp. 204-211. doi:10.1016/ S0040-6090(01) 01538-3

[28] N. Naghavi, D. Abou-Ras, N. Allsop, N. Barreau, S. Bücheler, A. Ennaoui, C. H. Fischer, C. Guillen, D. Hariskos, J. Herrero, R. Klenk, K. Kushiya, D. Lincot, R. Menner, T. Nakada, C. Platzer-Björkman, S. Spiering, a. N. Tiwari, T. Törndahl, Buffer layers and transparent conducting oxides for chalcopyrite $\mathrm{Cu}(\mathrm{In}, \mathrm{Ga})(\mathrm{S}, \mathrm{Se}) 2$ based thin film photovoltaics: Present status and current developments, Progress in Photovoltaics: Research and Applications 18 (6) (2010) 411-433. doi:10.1002/pip.955

[29] B. Vermang, J. T. Wätjen, V. Fjällström, F. Rostvall, M. Edoff, R. Kotipalli, F. Henry, D. Flandre, Employing Si solar cell technology to increase efficiency of ultra-thin $\mathrm{Cu}(\mathrm{In}, \mathrm{Ga}) \mathrm{Se} 2$ solar cells Progress in Photovoltaics: Research and Applications 22 (10) (2014) 1023-1029. doi:10.1002/pip.2527 URL http://dx.doi.org/10.1002/pip.1160http://doi. wiley.com/10.1002/pip.2527

[30] B. Vermang, V. Fjällström, J. Pettersson, P. Salomé, M. Edoff, Development of rear surface passivated $\mathrm{Cu}(\mathrm{In}, \mathrm{Ga}) \mathrm{Se} 2$ thin film solar cells with nano-sized local rear point contacts, Solar Energy Materials and Solar Cells 117 (2013) 505-511. doi:10.1016/j.solmat.2013.07.025

URL http://linkinghub.elsevier.com/retrieve/pii/ S0927024813003711

[31] M. A. Verschuuren, Substrate Conformal Imprint Lithography for Nanophotonics, Ph.D. thesis, Utrecht University (2010).

[32] M. Verschuuren, H. Sprang, 3D photonic structures by sol-gel imprint lithography, MRS Proceedings 1002 (mailstop 12) (2007) 1-6.

URL http://journals.cambridge.org/ abstract\{_\}S1946427400596456

[33] M. D. Kelzenberg, Appendix B: Silicon Microwire Photovoltaics, Ph.D. thesis, California Institute of Technology (2010).

[34] V. E. Ferry, Light trapping in plasmonic solar cells Ph.D. thesis, California Institute of Technology (2011).

URL http://www.ncbi.nlm.nih.gov/pubmed/20588593

[35] C. van Lare, F. Lenzmann, M. a. Verschuuren, A. Polman, Dielectric Scattering Patterns for Efficient Light Trapping in ThinFilm Solar Cells, Nano Letters (2015) 150630085420008doi: 10.1021/n15045583

URL http://pubs . acs . org/doi/abs/10.1021/nl5045583

[36] P. Wang, R. Menon, Optimization of periodic nanostructures for enhanced light-trapping in ultra-thin photovoltaics, Optics Express 21 (5) (2013) 6274. doi:10.1364/0E.21.006274

URL http://www.ncbi.nlm.nih.gov/pubmed/23482196https : //www.osapublishing.org/oe/abstract.cfm?uri= oe-21-5-6274

[37] Q. G. Du, C. H. Kam, H. V. Demir, H. Y. Yu, X. W. Sun, Enhanced optical absorption in nanopatterned silicon thin films with a nano-cone-hole structure for photovoltaic applications Optics Letters 36 (9) (2011) 1713. doi:10.1364/0L.36.001713 URL https://www.osapublishing.org/ol/abstract.cfm?uri= ol-36-9-1713

[38] A. Oskooi, P. a. Favuzzi, Y. Tanaka, H. Shigeta, Y. Kawakami, S. Noda, Partially disordered photonic-crystal thin films for enhanced and robust photovoltaics, Applied Physics Letters 100 (18) (2012) 181110. doi:10.1063/1.4711144 URL http://link.aip.org/link/APPLAB/v100/i18/p181110/ $\mathrm{s} 1\{\&\}$ Agg $=$ doi

[39] L. Ji, W. J. Nam, S. Fonash, Highly ordered nano-cone back reflector arrays for ultra-thin high performance CIGS cells in: 2013 IEEE 39th Photovoltaic Specialists Conference (PVSC), IEEE, 2013, pp. 1977-1979. doi:10.1109/PVSC.2013.6744858 URL http://ieeexplore.ieee.org/lpdocs/epic03/wrapper. htm?arnumber $=6744858$
[40] L. Yang, L. Mo, Y. Okuno, S. He, Optimal design of ultrabroadband, omnidirectional, and polarizationinsensitive amorphous silicon solar cells with a coreshell nanograting structure, Progress in Photovoltaics: ... (April 2012) (2013) 1077-1086. doi:10.1002/pip

URL http://onlinelibrary.wiley.com/doi/10.1002/pip. 2206/full 